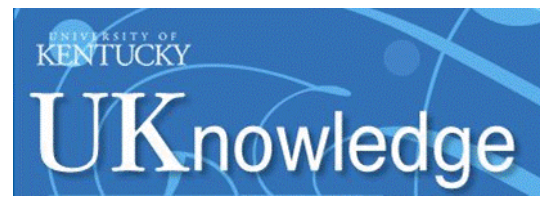

University of Kentucky

UKnowledge

\title{
Interplay of Composition, Structure, and Electron Density of States in W-Os Cathode Materials and Relationship with Thermionic Emission
}

Qunfei Zhou

University of Kentucky, zqfaye@gmail.com

Thomas John Balk

University of Kentucky, john.balk@uky.edu

Matthew J. Beck

University of Kentucky, beck@engr.uky.edu

Follow this and additional works at: https://uknowledge.uky.edu/cme_facpub

Part of the Chemical Engineering Commons, and the Materials Science and Engineering Commons Right click to open a feedback form in a new tab to let us know how this document benefits you.

\section{Repository Citation}

Zhou, Qunfei; Balk, Thomas John; and Beck, Matthew J., "Interplay of Composition, Structure, and Electron Density of States in W-Os Cathode Materials and Relationship with Thermionic Emission" (2017). Chemical and Materials Engineering Faculty Publications. 40.

https://uknowledge.uky.edu/cme_facpub/40

This Article is brought to you for free and open access by the Chemical and Materials Engineering at UKnowledge. It has been accepted for inclusion in Chemical and Materials Engineering Faculty Publications by an authorized administrator of UKnowledge. For more information, please contact UKnowledge@lsv.uky.edu. 


\title{
Interplay of Composition, Structure, and Electron Density of States in W-Os Cathode Materials and Relationship with Thermionic Emission
}

\author{
Digital Object Identifier (DOI) \\ https://doi.org/10.1116/1.4972857 \\ Notes/Citation Information \\ Published in Journal of Vacuum Science \& Technology A, v. 35, issue 2, 021601, p. 1-7. \\ (C) 2017 American Vacuum Society \\ This article may be downloaded for personal use only. Any other use requires prior permission of the \\ author and AIP Publishing. \\ The following article appeared in Journal of Vacuum Science \& Technology A, v. 35, issue 2, 021601, p. \\ 1-7 and may be found at https://doi.org/10.1116/1.4972857.
}




\title{
Interplay of composition, structure, and electron density of states in W-Os cathode materials and relationship with thermionic emission
}

\author{
Qunfei Zhou, Thomas John Balk, and Matthew J. Beck ${ }^{\text {a) }}$ \\ Department of Chemical and Materials Engineering, University of Kentucky, 177 F. Paul Anderson Tower, \\ Lexington, Kentucky 40506-0046
}

(Received 22 September 2016; accepted 9 December 2016; published 6 January 2017)

\begin{abstract}
The presence and composition of W-Os alloys have been found to significantly affect the thermionic emission properties of Os-coated tungsten dispenser cathodes. However, the comprehensive understanding of structure-property relationships needed to design improved tungsten cathodes with larger thermionic emission is still lacking. In this study, composition-structureproperty relationships governing thermionic emission from $\mathrm{W}-\mathrm{O}$ s alloys were investigated using quantum mechanical calculations. Low-energy W-Os atomic configurations at various compositions were determined from first-principles calculations based on density functional theory in combination with cluster expansion calculations. Electronic properties were investigated in terms of the electron density of states. The relative position of the Fermi level with respect to peaks and pseudogaps in the density of states for different W-Os structures can be used to explain, at least in part, observed variations in thermionic emission from Os-coated tungsten dispenser cathodes. (C) 2017 American Vacuum Society. [http://dx.doi.org/10.1116/1.4972857]
\end{abstract}

\section{INTRODUCTION}

Thermionic dispenser cathodes are electron emitting devices that produce high-current electron beams by thermally evaporating electrons from surfaces at temperatures typically $>1000{ }^{\circ} \mathrm{C}$. This is in contrast to field emission, or "cold," cathodes which leverage extremely high electric fields to strip electrons from the cathode. At such high operating temperatures, refractory metals - such as Os, Ru and W-with both high melting points ${ }^{1}$ and low vapor pressures ${ }^{2}$-are required to prevent rapid degradation of dispenser cathode emitting surfaces. The widely applied M-type dispenser cathode consists of a porous $\mathrm{W}$ pellet impregnated with a barium calcium aluminate compound, and then coated with an Os-Ru alloy layer that is crucial for achieving high emission current densities. ${ }^{3-7}$ M-type cathodes provide high emission current densities $\left(>10 \mathrm{~A} / \mathrm{cm}^{2}\right)$ at $\sim 1000^{\circ} \mathrm{C}$ under continuous duty operation. In contrast, impregnated W cathodes lacking the Os-Ru coating (called B-type cathodes) operate in the same temperature regime but at order-of-magnitude lower continuous duty currents $\left(1-4 \mathrm{~A} / \mathrm{cm}^{2}\right),{ }^{6}$ and in contrast to $\mathrm{LaB}_{6}$ or $\mathrm{CeB}_{6}$ cathodes which usually operate at higher temperatures of about $1400^{\circ} \mathrm{C}^{8,9}$

While the remarkable effect of Os/OsRu coatings on W cathodes has been widely investigated, the underlying mechanism of the effect remains unclear. It has been observed that variations in $\mathrm{W}$ content in the surface coatings have dramatic and nonlinear effects on emitted current density. ${ }^{5,6,11-14}$ As W and Os exhibit significant bulk miscibility (at least at high temperatures,${ }^{10}$ see Fig. 1), operation of M-type cathodes (typically at $1050^{\circ} \mathrm{C}$ ) results in increasing interdiffusion of $\mathrm{W}$ into the Os surface coating. ${ }^{15-17}$ Intriguingly, thermionic current density from Os-coated $\mathrm{W}$ cathodes first increases with

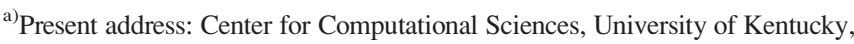
177 F. Paul Anderson Tower, Lexington, KY 40506; electronic mail: beck@engr.uky.edu
}

increasing $\mathrm{W}$ content at the cathode surface, then decreases dramatically [see, e.g., Fig. 2 (Ref. 5)]. Maximum emitted current density (minimum work function) at fixed temperature is achieved around $30-40$ at. $\% \mathrm{~W},{ }^{13,14}$ while at higher $\mathrm{W}$ concentrations, emitted current density is so low as to require cathode replacement. ${ }^{6,11,12}$ It should be noted that the spread in observed currents near the maximum $(\sim 40$ at. $\% \mathrm{~W})$ may reflect a coating thickness effect associated with varying $\mathrm{W}$ concentrations through the coating thickness. ${ }^{5}$

Previous studies have extensively examined the role that surface adsorbed $\mathrm{Ba}$ and/or $\mathrm{BaO}$ plays in reducing the apparent work function of cathode materials. ${ }^{18-22}$ For example, while the work function of pure $\mathrm{W}$ is $4.6 \mathrm{eV},{ }^{23} \mathrm{Ba} / \mathrm{BaO}$ adsorption at the surface of a W cathode lowers the work function to $\sim 2 \mathrm{eV}$ (and therefore increases emitted current density at fixed temperature). ${ }^{19}$ This effect is independent of, or in addition to, effects arising from the presence of an Os-Ru alloy coatings, as highlighted by the enhanced emission from M-type (coated) versus $\mathrm{B}$-type (uncoated) impregnated $\mathrm{W}$ cathodes. While $\mathrm{Ba}$ depletion would negate the effects of adsorbed $\mathrm{Ba} / \mathrm{BaO}$ (and thereby reduce cathode emission), $\mathrm{W}$ concentrations in the OsRu cathode coatings below $\sim 20$ at. $\%$ and above $\sim 80$ at. $\%$ have been shown to significantly reduce emitted current density even when sufficient $\mathrm{Ba}$ is present. ${ }^{15,17,24,25}$

Although both the high temperature phase diagram (see Fig. 1) and composition-dependent thermionic emission properties of W-Os alloys have been known for decades, the specific chemical and/or structural origins of compositiondriven changes in thermionic emission from W-Os alloys have remained largely unexplained. Previous studies have shown a correlation between the appearance of the W-Os $\sigma$ phase (a tetragonal phase) and decreased thermionic current density ${ }^{5}$ but key questions remain: Why does increasing $\mathrm{W}$ concentration in Os coatings increase thermionic emission for W concentrations below 30-40 at. \%, but not for higher $\mathrm{W}$ concentrations? Is it the appearance of the W-Os $\sigma$ phase 


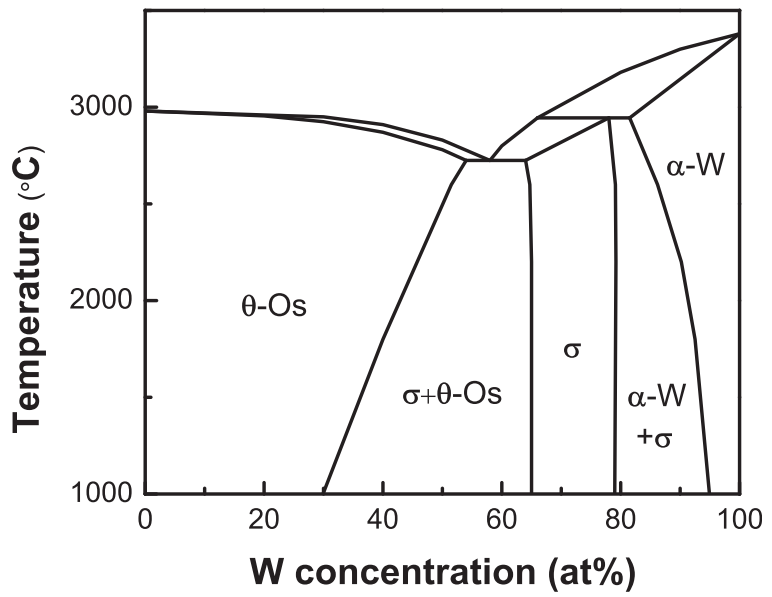

FIG. 1. Os-W phase diagram at temperatures above $1000^{\circ} \mathrm{C}$, adapted from Taylor et al., J. Less-Common Met. 3, 333 (1961).

that causes a dramatic reduction in thermionic current density at high $\mathrm{W}$ concentration? If so, why does the $\sigma$ phase exhibit lower emitted current densities?

Here, we apply quantum mechanical calculations based on density functional theory (DFT) in combination with cluster expansion-based (CE-based) methods to first deduce likely atomic arrangements present in W-Os alloys as a function of composition. We then use DFT to calculate the electronic structure of each atomic configuration. We find a subtle but significant difference between the electron density of states (eDOS) near the Fermi level for low W-concentration, highsymmetry HCP W-Os phases and higher W-concentration tetragonal phases. These findings suggest a chemical explanation for the observed changes in electron emission behavior of Os-coated W dispenser cathodes.

\section{METHODS}

Quantum mechanical calculations based on DFT are a standard and widely applied tool for computing materials structures and properties with little or no experimental input,

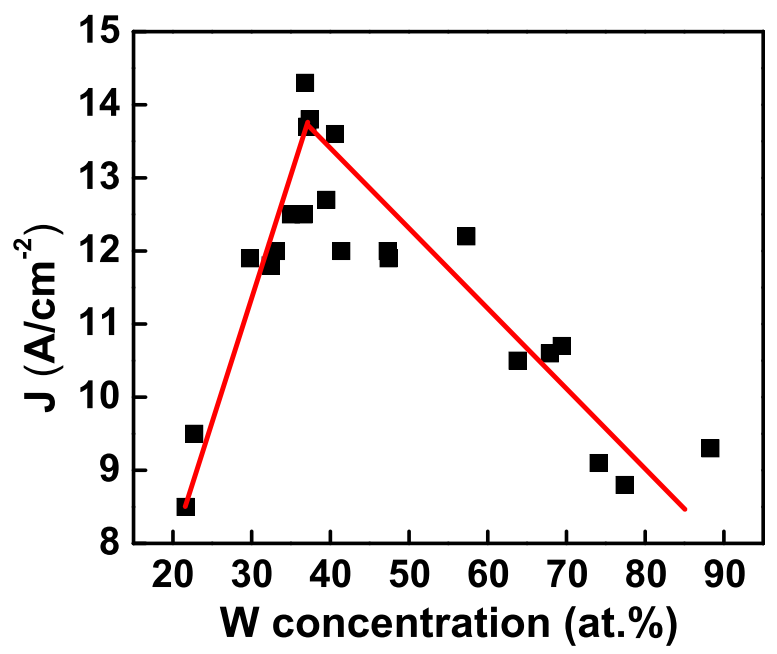

FIG. 2. (Color online) Current density $\mathrm{J}$ as a function of $\mathrm{W}$ composition in the coating layer, adapted from Brion et al., Appl. Surf. Sci. 20, 429 (1985). Lines are linear least squares fits to data above and below 37 at. \%. and have been extensively describe in the literature and textbooks (see, e.g., Refs. 26 and 27). Quantum mechanical calculations take as input simply the atomic numbers and initial positions of atoms in a system of interest. By solving the Schrödinger equation, quantum mechanical calculations compute the ground state arrangement of electrons for the given atomic positions. The computed charge density of electrons in space is then used to compute the Coulomb forces acting on atomic nuclei. Iterating these calculations allows the atomic positions to be "relaxed" to their lowest energy configuration, allowing direct determination of both the equilibrium structure and energy of systems of atoms. As these calculations solve the Schrödinger equation, resulting atomic structures and total energies account for the fully quantum mechanical nature of the electron/nuclei system. DFT-based methods, as used here, treat the electron-electron exchange-correlation potential using functionals of the charge density, which, while approximations, are well-controlled and widely studied, and have been applied to metal, semiconductor, insulator, and noncrystalline and molecular systems. ${ }^{28}$

In determining alloy structures, it is not computationally tractable to survey all possible atomic configurations of even one alloy system at one composition by using only DFT calculations. To address this limitation, an approach based on the cluster expansion ${ }^{29,30}$ — parameterized with quantum mechanical calculations-was used to screen possible W-Os alloy atomic configurations at compositions ranging from pure $\mathrm{W}$ to pure Os. This screening was done using the integrated alloy theoretic automated toolkit (ATAT), ${ }^{31,32}$ which allows efficient screening for ground state (minimum energy) atomic configurations of multicomponent alloys. ${ }^{33,34}$ A large number of previous studies have used this combined cluster expansion plus DFT calculations method to determine low-energy crystal structures, and have reported results in excellent agreement with experiments. These finding demonstrate the accuracy and robustness of the method as applied to a range of different materials systems, including metals, ${ }^{33-35}$ semiconductors, ${ }^{36,37}$ and complex layered structures. ${ }^{38}$

ATAT takes as input one or more parent crystal lattice types (e.g., HCP or BCC), and constructs possible atomic configurations at given concentrations by assigning different atomic species to lattice sites $(i)$. The energy $[\mathrm{E}(\sigma)]$ of these atomic configurations $(\sigma)$ is then computed as the sum over energies of configurational subunits ( $\alpha$, configurational "clusters" of atoms; typically pairs, triples, or quadruples of atoms), whose contributions to the total energy of a solid (effective interaction coefficients, $J_{\alpha}$ ) are fit from DFT calculations of the energy of a subset of structures, see Eq. (1) ${ }^{31,39}$

$$
E(\sigma)=\sum_{\alpha} m_{\alpha} J_{\alpha}\left\langle\prod_{i \in \alpha^{\prime}} \sigma_{i}\right\rangle,
$$

where $\alpha^{\prime}$ are subunits equivalent to $\alpha$ by symmetry and $m_{\alpha}$ is the number of $\alpha^{\prime}$. The set of all computed formation enthalpies for a given parent lattice (each for a different pattern of atoms on the lattice) are collected on an energy versus composition chart. The lowest energy structures at each composition are connected by a linear tie line, as in a phase diagram, 
to model two-phase composition regions, and the reduced set of lowest energy structures and tie-lines is called the "convex hull," and defines the set of all stable, ground-state phases of the alloy. Once the set of ground state alloy structures have been identified, DFT calculations are used to compute electronic properties.

To increase accuracy of ATAT calculations for complex alloys, and to account for chemical differences (including, e.g., coordination effects) among lattice types, ATAT computes independent effective interaction coefficients for cluster expansions based on different lattice types. Based on the experimental phase diagram for $\mathrm{W}-\mathrm{O}$ s and the crystal structures of pure $\mathrm{W}(\mathrm{BCC})$ and $\mathrm{Os}(\mathrm{HCP})$, here we have considered configurations arising from $\mathrm{BCC}$ and $\mathrm{HCP}$ unit cells. To measure the accuracy of CE-computed formation enthalpies, and to validate the fitted effective interaction coefficients, ATAT compares CE-computed formation enthalpies to DFT-computed values via the statistical cross-validation technique. ${ }^{31}$ The final cross-validation scores-which represent the estimated error in the cluster-expansion computed enthalpies-for the calculations of HCP- and BCC-based configurations reported here are 0.018 and $0.024 \mathrm{eV} /$ atom, respectively.

All DFT calculations reported here, for both low-energy structure calculations combined with cluster expansion in ATAT and electronic structure calculations, were performed using the commercially available Vienna ab initio simulation package. ${ }^{40,41}$ Formation enthalpies were obtained from static calculations using GGA exchange-correlation functionals. ${ }^{42}$ Low-energy structures used as inputs to calculations of formation enthalpies were obtained from relaxation calculations using LDA exchange-correlation functionals. ${ }^{43}$ The $k$-point densities and energy cutoffs have been tested to give numerical convergence of DFT results to $1 \mathrm{meV} /$ atom. The density of special $k$-points used to compute electronic wavefunctions was specified in terms of the number of $k$-points per reciprocal atom, ${ }^{31}$ which were 1500 and 4100 for calculations on HCP- and BCC-based structures, respectively. The planewave energy cutoff was 334 and $387 \mathrm{eV}$ for, again, HCPand BCC-based structures, respectively.

To enable direct comparisons of formation enthalpies for configurations with different numbers of Os and $\mathrm{W}$ atoms, a fixed energy reference must be chosen. Here we take as reference energies that of an Os atom in pure HCP Os, and of a $\mathrm{W}$ atom in pure BCC W. Calculations of the eDOS using DFT are well-established and have been widely used in various systems including metals and semiconductors. ${ }^{26}$ The plane-wave energy cutoffs for eDOS calculations are the same as those for calculations of formation enthalpies, while the $k$-point sampling was performed using Monkhorst-Pack mesh grids of about $30 \times 30 \times 30$.

As a final note, ATAT calculations seeking low-energy alloy configurations required in excess of 20000 core-hours and considered $\sim 1000$ distinct W-Os configurations over a range of $\mathrm{W}$ concentrations. DFT calculations for final relaxed structures and eDOS of low-energy, reference and cluster expansion fitting configurations required up to an additional 400 core-hours per configuration. Calculations were primarily completed on the Lipscomb Supercomputing Cluster at the University of Kentucky.

\section{RESULTS AND DISCUSSION}

\section{A. Stable W-Os alloy structures}

While the experimentally determined W-Os phase diagram shows the expected phases of W-Os alloys as a function of composition, calculation of the electronic structure requires knowledge of the specific atomic arrangements within each phase. ATAT was used to search for low-energy atomic arrangements based on both BCC and HCP lattices. Figure 3 shows the formation enthalpies of all $\sim 1000$ alloy configurations calculated in this study. Each plotted point indicates the formation enthalpy of a particular atomic configuration with a particular composition. Data points marked as crosses and stars are formation enthalpies of configurations with HCP based lattice structures, while triangles are formation enthalpies for configurations with BCC based lattice structures. The predicted formation enthalpies were computed based on the cluster expansion alone, while the fitted formation enthalpies represent results from DFT calculations. The cluster expansion effective interaction coefficients were fit independently for each lattice type to DFTcalculated enthalpies. The solid red and blue lines sketch the convex hull of enthalpies for BCC- and HCP-based structures, respectively, and highlight low-energy atomic structures for each set. The overall convex hull is indicated with a solid black line.

Figure 4 shows the primitive unit cells of the five lowenergy HCP-based atomic structures identified for W concentrations less than 70 at. \%, plus the one low-energy BCC-based structure with $\mathrm{W}$ concentration greater than 70 at. \%, along with those of pure HCP Os and BCC W. Table I reports the DFT-calculated structural parameters of the six atomic configurations shown in Fig. 4. Previous experimental determinations of crystal structure and lattice

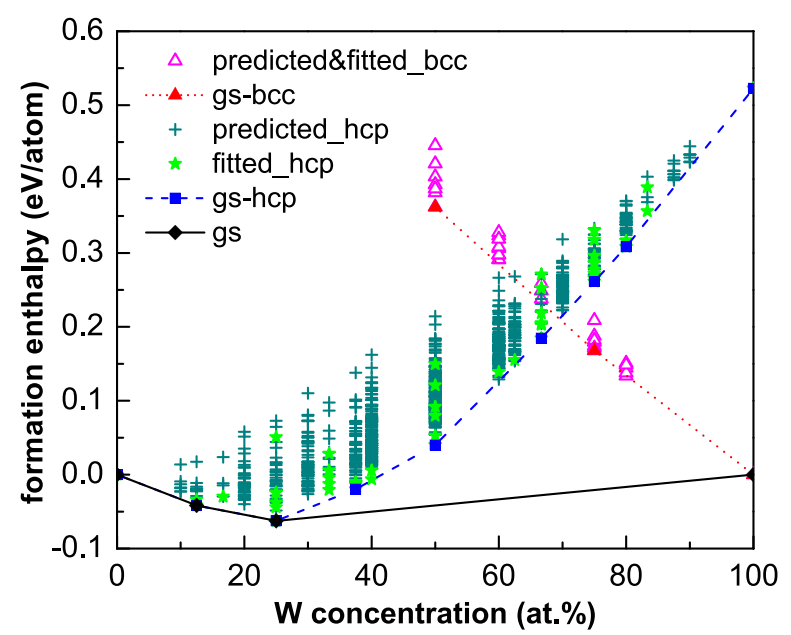

FIG. 3. (Color online) Formation enthalpies as a function of W concentration for all the calculated W-Os configurations with various atomic arrangements. The dashed and dotted convex hulls are constructed from HCP and BCC-based configurations, respectively. The solid curve is the absolute convex hull connecting the two ground-state alloys. 

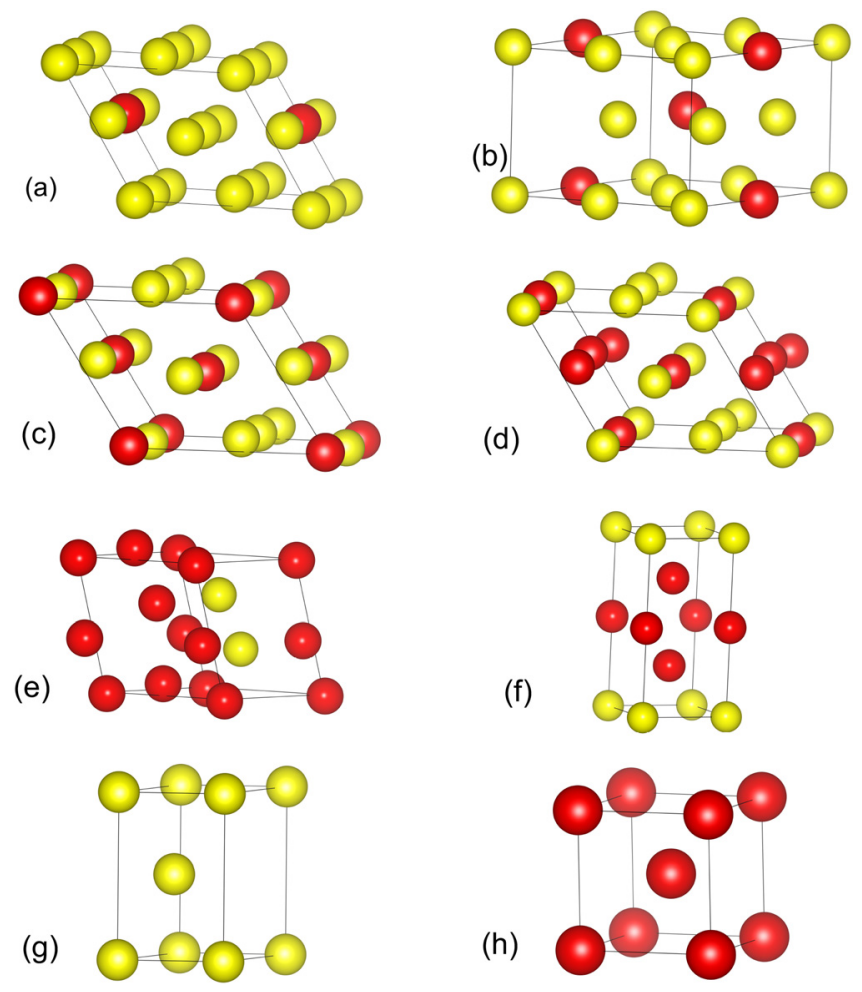

FIG. 4. (Color online) Primitive cells of the predicted stable structures, with $\mathrm{W}$ atoms a little larger than Os atoms. The crystal structure type is represented in the form of chemical-formula.Pearson-symbol(space group). (a) $\mathrm{WOs}_{7} \cdot \mathrm{oF} 32(\mathrm{Fm} 2 \mathrm{~m})$; (b) $\mathrm{WOs}_{3} \cdot \mathrm{hP} 8\left(\mathrm{P}_{3} / \mathrm{mmc}\right.$ ); (c) $\mathrm{W}_{3} \mathrm{Os}_{5} \cdot \mathrm{oF} 32(\mathrm{Fm} 2 \mathrm{~m})$; (d) WOs.oF32(F2dd); (e) $\mathrm{W}_{2} \mathrm{Os} \cdot \mathrm{mC} 12(\mathrm{C} 2 / \mathrm{m})$; (f) $\mathrm{W}_{3} \mathrm{Os} \cdot \mathrm{PP} 4(\mathrm{P} 4 / \mathrm{mmm})$; (g) $\mathrm{W}_{3} \mathrm{Os} \cdot \mathrm{hP} 2\left(\mathrm{P}_{3} / \mathrm{mmc}\right)$; and $(\mathrm{h}) \mathrm{W} \cdot \operatorname{cI} 2(\operatorname{Im} \overline{3} \mathrm{~m})$.

parameters are available for a number of alloy compositions close to those of five of the low-energy alloy structures. In these experimental studies, $\mathrm{x}$-ray and micrographic techniques were used to determine the phase and lattice parameters of W-Os alloys rapidly quenched from $2400{ }^{\circ} \mathrm{C}$. As can be seen in Table I, excellent agreement between computed and measured properties is observed for W-Os compositions except those with greater than 50 at. \% W. While experimentally determined lattice parameters are not available for the 50 at. $\% \mathrm{~W}$ alloy, the crystal structure has been experimentally identified as orthorhombic $\mathrm{AuCd},{ }^{44}$ the same as found here.

Based on formation energies reported in Fig. 3 (see the black overall convex hull), the W-Os system is predicted to have only two absolute ground state alloy structures (the HCP-related face-centered orthorhombic $\mathrm{WOs}_{7}$, and hexagonal $\mathrm{WOs}_{3}$ ) plus the pure component end points. In contrast, the experimental W-Os phase diagram shows no intermediate $\mathrm{HCP}$ or face-centered orthorhombic alloy phases, but, instead, a single tetragonal $\sigma$ phase that is stable at $\mathrm{W}$ concentrations greater than $\sim 60$ at. $\% \mathrm{~W}$. This corresponds to the composition region where agreement between computed and measured lattice parameters falters. The present calculations do, in fact, identify a tetragonal structure $\left(\mathrm{W}_{3} \mathrm{Os}\right)$ that is the lowest-energy BCC-family structure, but its computed formation enthalpy is well above that predicted for a two-phase system consisting of pure BCC W and hexagonal $\mathrm{WOs}_{3}$ (see black convex hull in Fig. 3). Previous calculations screening for W-Os ground state structures using a similar methodology as the present work have identified a tetragonal structure with a large unit cell, tP30 $\left(\mathrm{W}_{22} \mathrm{Os}_{8}\right)$, that has lower formation energy than both tetragonal $\mathrm{W}_{3} \mathrm{Os}$ and the two-phase $\mathrm{BCC} \mathrm{W}$ plus hexagonal $\mathrm{WOs}_{3}$ alloy. ${ }^{46}$ Computed structural properties of this $\mathrm{W}_{22} \mathrm{Os}_{8}[73.3$ at. \% W, see Fig. 5(a)] tP30 structure give excellent agreement with experimentally measured properties of the W-Os $\sigma$ phase (see Table I).

Considering the computationally predicted intermediate HCP and face-centered orthorhombic phases, we note that the HCP and face-centered orthorhombic primitives identified here differ only by the symmetry of their arrangement of $\mathrm{W}$ and Os atoms. That is, they all exhibit close-packed HCP layer stacking by atomic positions, but the pattern of which atomic positions are occupied by $\mathrm{W}$ and Os breaks the $\mathrm{AB}$ stacking symmetry. More generally we note that the HCP, FCC, and orthorhombic primitives relevant here can all be constructed from the same arrangement of atomic positions [see Fig. 5(b)]. In a two component alloy like W-Os, the ordering of $\mathrm{W}$ and Os on specific atomic sites will determine which symmetries are retained, and what the primitive cell is for the structure. If atom types were to be ignored, all of the structures in Fig. 4 would have an HCP primitive (maximum symmetry), but accommodating which atomic positions are $\mathrm{W}$ and which are Os yields first FCC, then orthorhombic primitives as symmetry is broken.

In this way, at finite temperature, where $\mathrm{W}$ and Os atoms will exhibit thermally induced intermixing on the crystal lattice sites, time and space averaged measurements of atomic

TABLE I. Comparison of measured and computed structural parameters for the W-Os alloys discussed here. Note: the experimental data for the $\sigma$-tP30 phase (at $\mathrm{T} \geq 1000^{\circ} \mathrm{C}$ ) are from Ref. 45 . All the other experimental information is from Ref. 10 .

\begin{tabular}{|c|c|c|c|c|c|c|c|}
\hline \multicolumn{2}{|l|}{ Exp. } & \multicolumn{2}{|c|}{ This study } & \multicolumn{2}{|c|}{ Lattice constant $(\AA)$} & \multicolumn{2}{|c|}{ c/a ratio } \\
\hline at. $\% \mathrm{~W}$ & Phase & at. $\% \mathrm{~W}$ & Structure & Exp. & This study & Exp. & This study \\
\hline 10 & $\theta$ & 12.5 & $\mathrm{oF} 32$ & $a=2.74, c=4.34$ & $a=2.73, c=4.30$ & 1.5825 & 1.5762 \\
\hline 30 & $\theta$ & 25 & hP8 & $a=2.76, c=4.41$ & $a=2.74, c=4.35$ & 1.5961 & 1.5901 \\
\hline 40 & $\sigma+\theta$ & 37.5 & $\mathrm{oF} 32$ & $a=2.76, c=4.45$ & $a=2.75, c=4.40$ & 1.6090 & 1.5986 \\
\hline 50 & $\sigma+\theta$ & 50 & $\mathrm{oF} 32$ & - & $a=2.79, c=4.52$ & - & 1.6223 \\
\hline 66.7 & $\sigma$ & 66.7 & $\mathrm{mC} 12$ & $a=9.63, c=4.98$ & $a=10.33, c=5.55$ & 0.5170 & 0.5378 \\
\hline 75 & $\sigma$ & 75 & tP4 & $a=9.66, c=5.01$ & $a=6.20, c=3.11$ & 0.5181 & 0.5021 \\
\hline $64-80$ & $\mathrm{tP} 30$ & 73.3 & tP30 & $a=9.65, c=4.99$ & $a=9.70, c=4.99$ & 0.5171 & 0.5152 \\
\hline
\end{tabular}


(a)

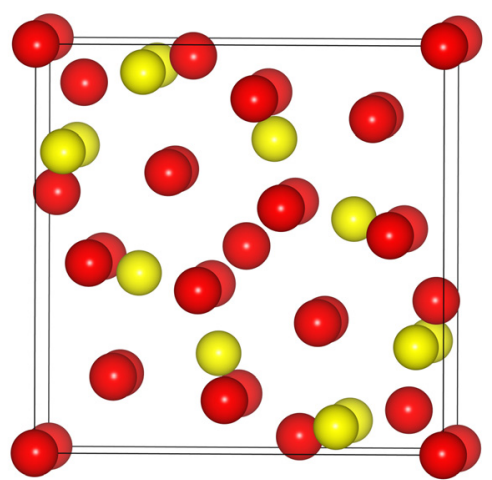

(b)

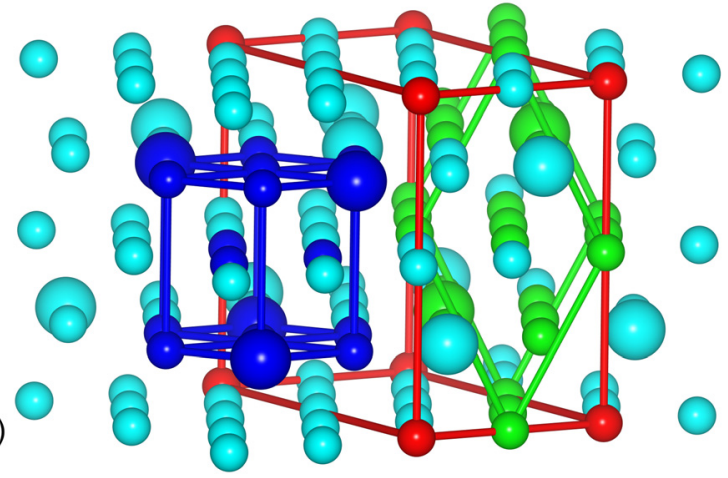

FIG. 5. (Color online) Atomic arrangement of $\mathrm{W}_{22} \mathrm{Os}_{8} \cdot \mathrm{tP} 30\left(\mathrm{P}_{2} / \mathrm{mnm}\right)(\mathrm{a})$; The standard unit cell (orthorhombic), primitive cell (parallelepiped) and hexagonal cell drawn for the oF32 WOs $\mathrm{W}_{7}$ crystal lattice. The size of W atoms has been enlarged in order to distinguish them from Os atoms.

structure (via, e.g., XRD) will see increased symmetry compared to the computed instantaneous ground state structures as lattice sites become indistinguishable from each other due to $\mathrm{W}$ and $\mathrm{Os}$ site switching. We note that in all $\mathrm{HCP}$ and face-centered orthorhombic structures considered here both the $\mathrm{W}$ and $\mathrm{Os}$ atoms are 12-fold coordinated. Therefore, the energy penalty for site exchange-related disorder in these structures is expected to be low, and at the temperatures relevant for the $\mathrm{W}$-Os phase diagram $\left(\geq 1000^{\circ} \mathrm{C}\right)$ the symmetry destroying ordering of $\mathrm{W}$ and Os on crystal lattice sites is likely not observed: hence, ground state calculations predict a series of specific HCP-family phases, while experiment observes a single solid solution HCP $\theta$ phase for low $\mathrm{W}$ concentrations in Os.

In contrast, in BCC-based structures (like tP30), W atoms exhibit a range of coordination states, with some $\mathrm{W}$ atoms having as few as five coordinating neighbors. Os atoms remain 12 -fold coordinated. This implies that exchanging $\mathrm{W}$ and Os requires overcoming a larger energy penalty than exchanges on HCP-based lattices, and highlights the sensitivity of the overall formation enthalpy of BCC-based structures to details of atomic ordering. This likely explains why the $\sigma$-phase requires a large primitive cell that is not well resolved in the present cluster expansion calculations.

\section{B. Electronic properties}

Having determined detailed atomic arrangements relevant to W-Os alloys in thermionic cathodes, we now turn to assessing the electronic properties of these alloys. Thermionic current density is described by the RichardsonDushman equation $^{47,48}$

$$
J=A_{R} T^{2} \exp \left(-\frac{\Phi}{k_{B} T}\right),
$$

in terms of the energy barrier to electron emission (as characterized by the work function, $\Phi)$, the operating temperature $(T)$, and a materials-specific prefactor $\left(A_{R}\right)$ characterizing the distribution in energy of electrons available to be emitted-that is, the electronic density of states (eDOS) in the emitting material. In its original form, Richardson took $A_{R}$ to be a constant with the value of $120 \mathrm{~A} \mathrm{~cm}^{-2} \mathrm{~K}^{-2}$, which is computed for the eDOS of the free electron gas. The value of $A_{R}$, though, is highly material dependent, as has been discussed in theoretical studies ${ }^{48,49}$ and shown in experimental reports (fitted from $\mathrm{J}-\Phi$ curves) where $A_{R}$ differs by orders of magnitude. ${ }^{13,48}$

While the work function plays a critical role in determining emitted thermionic current density at a given temperature, the role of the emitting material's electronic density of states - as captured in the material-dependent value of $A_{R}$ is nontrivial. The work function is defined as the energy barrier for an electron at the Fermi level to be evaporated into the vacuum. Electrons bound in the crystal at energies below the Fermi level may also be thermally evaporated, but must overcome an energy barrier equal to the work function plus the amount of energy below the Fermi level at which they are bound. Different materials have different densities of electrons at and near the Fermi level implying different energy-weighted numbers of electrons available to be thermally excited to vacuum states. Therefore variations in eDOS (particularly near the Fermi level) can have important consequences for the emitted current density at a given temperature that are in addition to effects due to differences in work function.

Computed eDOS for low-energy W-Os alloy configurations are shown in Fig. 6, with the Fermi level indicated for each with a dashed blue line. All considered alloys exhibit qualitatively similar eDOS containing a pseudogap (region in energy of low DOS) near the Fermi level-a feature typical of many transition metals or alloys. ${ }^{50}$ The position of the Fermi level relative to the pseudogap, though, varies between different W-Os configurations. Similar variations in other alloy systems have been observed, and have been related to differences in a range of bulk properties, including structure stabilities and melting point (low DOS at the Fermi level has been reported to correlate with high structure stability and high melting point). ${ }^{50,51}$

Here, we are particularly concerned with the total eDOS at or just below the Fermi Level, as these "near-Fermi" electrons represent the dominant contributors to the set of electrons thermally emitted from W-Os surfaces during thermionic emission, as discussed above. As shown in Figs. 6(a)-6(e), for pure HCP Os and $\mathrm{WOs}_{7}$, the Fermi level sits in the middle of the pseudogap and moves close to the left edge of the pseudogap for $\mathrm{WOs}_{3}, \mathrm{~W}_{3} \mathrm{Os}_{5}$, and WOs. For 


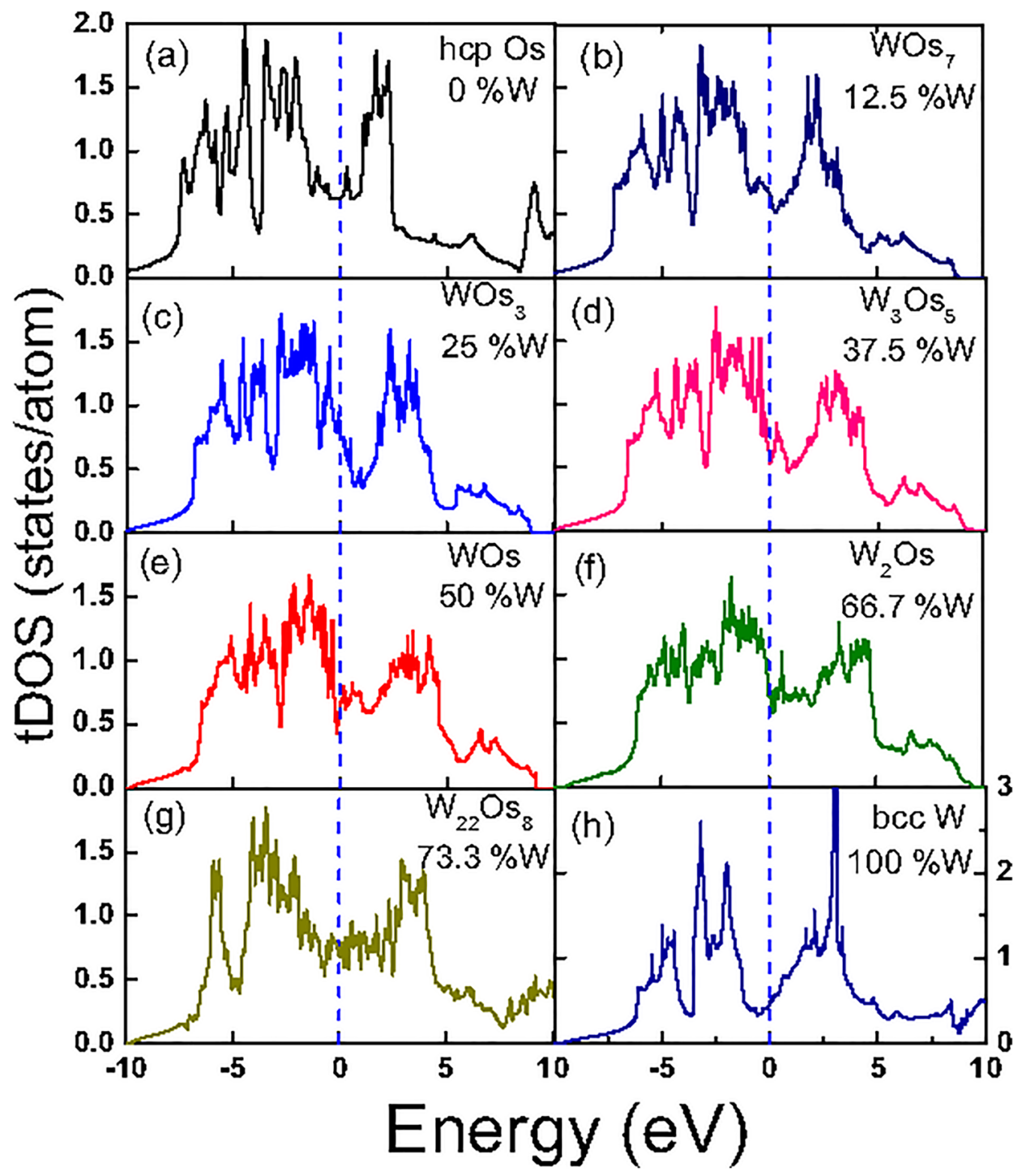

FIG. 6. (Color online) Electron density of states of HCP Os (a), the predicted W-Os alloy ground-state structures (b)-(f), tP30 (g) and BCC W (h). The vertical dashed lines are the positions of the Fermi level.

$\mathrm{W}_{2} \mathrm{Os}$ with 66.7 at. $\% \mathrm{~W}$, the Fermi level sits right at the edge of the pseudogap with high DOS. For $\mathrm{PP}^{\mathrm{P}} 3 \mathrm{~W}_{22} \mathrm{Os}_{8}$, the Fermi level lies inside the pseudogap, similar to HCP Os, $\mathrm{WOs}_{7}$, and BCC W.

The high DOS just below the Fermi level for $\mathrm{WOs}_{3}$, $\mathrm{W}_{3} \mathrm{Os}_{5}$, and WOs indicates that larger numbers of electrons are available to be thermionically emitted at a given temperature than for $\mathrm{HCP} \mathrm{Os}_{\mathrm{N}} \mathrm{WO}_{7}, \mathrm{BCC} \mathrm{W}$, and $\mathrm{tP}_{3} \mathrm{~W}_{22} \mathrm{Os}_{8}$, which all have much lower DOS just below the Fermi level. That is, from the perspective of how many electrons are available with energies near the Fermi level (and therefore with energy barriers to emission equal to or trivially greater than the work function, see discussion above), thermionic current from W-Os alloys should first increase with increasing $\mathrm{W}$ concentration $\left(\mathrm{HCP}\right.$ Os and $\mathrm{WO}_{7}$ to $\mathrm{WOs}_{3}, \mathrm{~W}_{3} \mathrm{Os}_{5}$, and WOs), then decrease ( $\mathrm{WOs}_{3}, \mathrm{~W}_{3} \mathrm{Os}_{5}$, and WOs to tP30 $\mathrm{W}_{22} \mathrm{Os}_{8}$ and $\mathrm{BCC} \mathrm{W}$ ), exactly as observed experimentally (see Fig. 2).

Returning to the key questions identified at the beginning of this paper, we note that the present results suggest that differences in eDOS at or just below the Fermi level have an important impact on the variation of thermionic emission with composition. The high eDOS just below the Fermi level for 12-fold coordinated HCP-based alloy phases $\mathrm{WOs}_{3}$, $\mathrm{W}_{3} \mathrm{Os}_{5}$, and WOs corresponds to experimental observations of enhanced thermionic emission at moderate W-concentrations in Os coatings, while the lower eDOS at or just below the Fermi level of W-Os phases with higher or lower W-concentration correspond to experimental observations of lower thermionic emission. Thus, increasing the stability of high W-content HCP phases of W-Os alloys could increase the lifetime of thermionic cathodes by delaying the onset of emission reduction leading to end-of-life of cathode.

\section{CONCLUSION}

Stable atomic configurations of W-Os alloys have been obtained by combining DFT calculations and the cluster expansion for both BCC- and HCP-family lattices. Computed structural parameters are very close to experimentally 
determined values. Differences in atomic structure between $\mathrm{HCP}$, tetragonal, and $\mathrm{BCC} \mathrm{W}-\mathrm{Os}$ phases alter the position of the Fermi level relative to a pseudogap in the W-Os electron density of states. When the Fermi level is at the left edge of the pseudogap, and therefore there is high eDOS just below the Fermi level—as for high W-content HCP phases-thermionic emission is expected to be enhanced. In contrast, when the Fermi level is located near the top of the pseudogap-as for tetragonal and BCC W-Os phases-low near-Fermi electron density suggests weaker thermionic emission. These composition driven changes in electronic structure can explain previously observed changes in dispenser cathode emission. This suggests that thermionic cathode lifetime could be extended by stabilizing high-W content HCP phases, which are expected to exhibit stronger thermionic emission. Finally, the methods used in this work can also be applied to other material systems, though only W-Os alloys have been discussed here. We note that the eDOS is a readily computed property that can be used in qualitative evaluation of thermionic electron emission for cathode materials.

\section{ACKNOWLEDGMENTS}

This material is based upon work supported by the National Science Foundation under Grant No. CMMI0928845. The authors give special thanks to Phillip D. Swartzentruber for the discussion and help on experimental insight of thermionic emission from Os-coated tungsten dispenser cathode.

${ }^{1}$ J. Arblaster, Platinum Met. Rev. 49, 166 (2005).

${ }^{2}$ M. B. Panish and L. Reif, J. Chem. Phys. 37, 128 (1962).

${ }^{3}$ P. Zalm and A. J. A. van Stratum, Philips Tech. Rev. 27, 69 (1966).

${ }^{4}$ M. Green, H. Skinner, and R. Tuck, Appl. Surf. Sci. 8, 13 (1981).

${ }^{5}$ D. Brion, J.-C. Tonnerre, and A. Shroff, Appl. Surf. Sci. 20, 429 (1985).

${ }^{6}$ A. Shih, A. Berry, C. R. K. Marrian, and G. A. Haas, IEEE Trans. Electron Devices 34, 1193 (1987).

${ }^{7}$ J. Cronin, IEE Proc I 128, 19 (1981).

${ }^{8}$ K. Togawa, T. Shintake, T. Inagaki, K. Onoe, T. Tanaka, H. Baba, and H. Matsumoto, Phys. Rev. Spec. Top-Accel. 10, 020703 (2007).

${ }^{9}$ A. Taran, D. Voronovich, S. Plankovskyy, V. Paderno, and V. Filipov, IEEE Trans. Electron Devices 56, 812 (2009).

${ }^{10}$ A. Taylor, B. Kagle, and N. Doyle, J. Less-Common Met. 3, 333 (1961).

${ }^{11}$ R. Thomas and J. Gibson, Appl. Surf. Sci. 29, 49 (1987).

${ }^{12}$ P. D. Swartzentruber, T. John Balk, and S. Roberts, Proceedings of the 2011 IEEE International Vacuum Electronics Conference (IVEC) (2011), pp. 401-402.

${ }^{13}$ P. D. Swartzentruber, T. John Balk, and M. P. Effgen, J. Vac. Sci. Technol., A 32, 040601 (2014).

${ }^{14}$ P. D. Swartzentruber, M. J. Detisch, and T. J. Balk, J. Vac. Sci. Technol., A 33, 021405 (2015).
${ }^{15}$ L. R. Falce, Proceedings of the 2004 International Vacuum Electronics Conference (IVEC) (2004), pp. 356-357.

${ }^{16}$ N. Mita, IEEE Trans. Electron Devices 38, 2554 (1991).

${ }^{17}$ T. Balk, W. Li, and S. Roberts, Proceedings of the 2009 IEEE International Vacuum Electronics Conference (IVEC) (2009), pp. 177-178.

${ }^{18}$ D. S. Chen, I. Lindau, M. H. Hecht, and A. J. Viescas, Appl. Surf. Sci. 13, 321 (1982).

${ }^{19}$ W. Müller, IEEE Trans. Electron Devices 36, 180 (1989).

${ }^{20}$ R. Cortenraad, A. W. Denier van der Gon, H. H. Brongersma, G. Gärtner, and A. Manenschijn, Appl. Surf. Sci. 191, 153 (2002).

${ }^{21}$ Z. A. Ibrahim and M. J. G. Lee, Phys. Rev. B 76, 155423 (2007).

${ }^{22}$ M. C. Lin, R. F. Jao, and W. C. Lin, J. Vac. Sci. Technol., B 26, 821 (2008).

${ }^{23}$ D. R. Lide, CRC Handbook of Chemistry and Physics, 85th ed. (Taylor \& Francis, Boca Raton, FL, 2004), pp. 12-130.

${ }^{24}$ W. C. Li, S. Roberts, and T. J. Balk, IEEE Trans. Electron Devices 56, 805 (2009).

${ }^{25}$ R. E. Thomas, J. W. Gibson, G. A. Haas, and R. H. Abrams, Jr., IEEE Trans. Electron Devices 37, 850 (1990).

${ }^{26}$ D. Sholl and J. A. Steckel, Density Functional Theory: A Practical Introduction (John Wiley \& Sons, Inc, Hoboken, NJ, 2011), pp. 179-188.

${ }^{27}$ R. M. Martin, Electronic Structure: Basic Theory and Practical Methods (Cambridge University, Cambridge, UK, 2004).

${ }^{28}$ J. Hafner, Comput. Phys. Commun. 177, 6 (2007).

${ }^{29}$ D. B. Laks, L. G. Ferreira, S. Froyen, and A. Zunger, Phys. Rev. B 46, 12587 (1992).

${ }^{30}$ J. Sanchez, F. Ducastelle, and D. Gratias, Physica A 128, 334 (1984).

${ }^{31}$ A. van de Walle, M. Asta, and G. Ceder, Calphad 26, 539 (2002).

${ }^{32}$ A. De van Walle and G. Ceder, J. Phase Equilib. 23, 348 (2002).

${ }^{33}$ R. H. Taylor, S. Curtarolo, and G. L. W. Hart, Phys. Rev. B 81, 024112 (2010).

${ }^{34}$ S. Curtarolo, D. Morgan, and G. Ceder, Calphad 29, 163 (2005).

${ }^{35}$ C. Ravi, B. Panigrahi, M. Valsakumar, and A. van de Walle, Phys. Rev. B 85, 054202 (2012).

${ }^{36}$ Y. Zhang, E. Skoug, J. Cain, V. Ozolinšs, D. Morelli, and C. Wolverton, Phys. Rev. B 85, 054306 (2012).

${ }^{37}$ B. Burton, S. Demers, and A. Van de Walle, J. Appl. Phys. 110, 023507 (2011).

${ }^{38}$ K. Govaerts, M. Sluiter, B. Partoens, and D. Lamoen, Phys. Rev. B 85, 144114 (2012).

${ }^{39}$ V. Blum and A. Zunger, Phys. Rev. B 70, 155108 (2004).

${ }^{40}$ G. Kresse and J. Hafner, Phys. Rev. B 47, 558 (1993).

${ }^{41}$ G. Kresse and J. Furthmuller, Phys. Rev. B 54, 11169 (1996).

${ }^{42}$ J. P. Perdew, K. Burke, and M. Ernzerhof, Phys. Rev. Lett. 77, 3865 (1996).

${ }^{43}$ D. M. Ceperley and B. J. Alder, Phys. Rev. Lett. 45, 566 (1980).

${ }^{44}$ R. Coehoorn, G. V. D. Kolk, J. V. D. Broek, T. Minemura, and A. Miedema, J. Less-Common Met. 140, 307 (1988).

${ }^{45}$ P. R. Rao, Phase Diagrams of Binary Tungsten Alloys (ASM Intl., OH, 1991), Vol. 7.

46"Automatic-flow for materials discovery," http://aflowlib.org/advanced.php.

${ }^{47} \mathrm{O}$. W. Richardson, The Emission of Electricity from Hot Bodies (Longmans, Green and Company, London, UK, 1921).

${ }^{48}$ S. Dushman, Rev. Mod. Phys. 2, 381 (1930).

${ }^{49}$ C. R. Crowell, Solid State Electron. 8, 395 (1965).

${ }^{50}$ T. Hong, T. Watson-Yang, X.-Q. Guo, A. Freeman, T. Oguchi, and J.-H. Xu, Phys. Rev. B 43, 1940 (1991).

${ }^{51}$ P. Ravindran and R. Asokamani, Bull. Mater. Sci. 20, 613 (1997). 


\section{Interplay of composition, structure, and electron density of states in W-Os cathode materials and relationship with thermionic emission}

Qunfei Zhou, Thomas John Balk, and Matthew J. Beck

Citation: Journal of Vacuum Science \& Technology A 35, 021601 (2017); doi: 10.1116/1.4972857

View online: https://doi.org/10.1116/1.4972857

View Table of Contents: http://avs.scitation.org/toc/jva/35/2

Published by the American Vacuum Society

\section{Articles you may be interested in}

Challenges in atomic layer deposition of carbon-containing silicon-based dielectrics Journal of Vacuum Science \& Technology A: Vacuum, Surfaces, and Films 35, 021506 (2017); $10.1116 / 1.4973923$

Rarefied gas flow into vacuum through short tubes at variable wall temperatures Journal of Vacuum Science \& Technology A: Vacuum, Surfaces, and Films 35, 021604 (2017); $10.1116 / 1.4974751$

Effect of reactor pressure on the conformal coating inside porous substrates by atomic layer deposition Journal of Vacuum Science \& Technology A: Vacuum, Surfaces, and Films 35, 021502 (2017); $10.1116 / 1.4973350$

Contrasting transparent conductive properties of $\mathrm{ZnO}$ films on amorphous and crystalline substrates in view of thickness dependence Journal of Vacuum Science \& Technology A: Vacuum, Surfaces, and Films 35, 021503 (2017); $10.1116 / 1.4973540$

In situ absolute magnetometry in an UHV scanning probe microscope using conducting polymer-thin film Journal of Vacuum Science \& Technology A: Vacuum, Surfaces, and Films 35, 021602 (2017); $10.1116 / 1.4973920$

$A b$ initio determination of electron affinity of polar nitride surfaces, clean and under Cs coverage Journal of Vacuum Science \& Technology A: Vacuum, Surfaces, and Films 35, 021406 (2017); $10.1116 / 1.4975332$

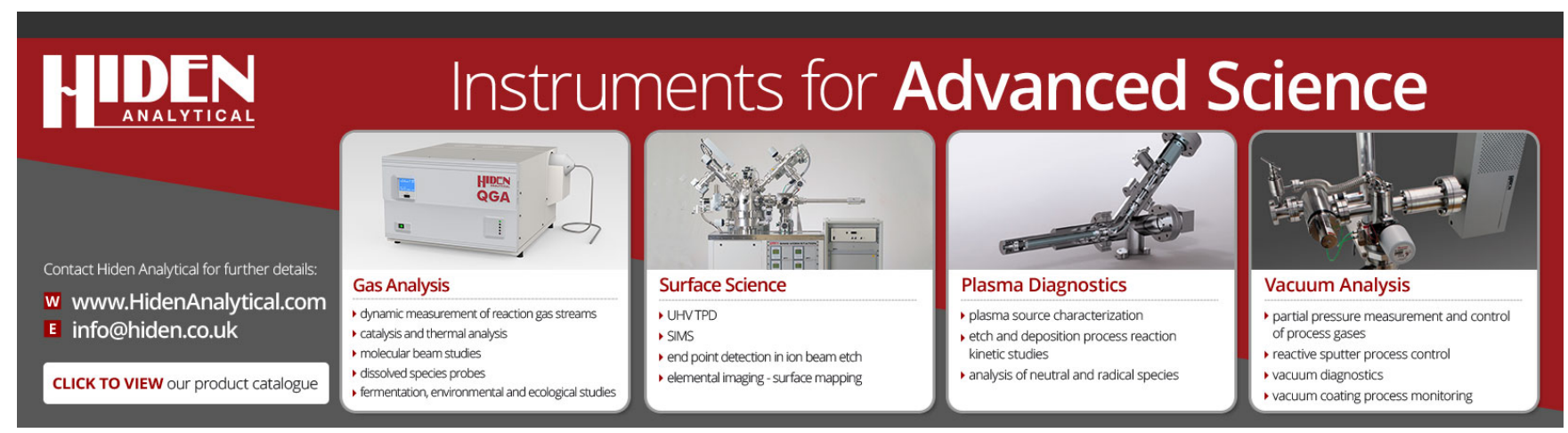

